\title{
In vitro regeneration protocol for indica rice genotypes
}

\author{
Kaium Miah ${ }^{1}$, Billal Hossen ${ }^{1}$, M. Shahidul Haque ${ }^{2}$, M. Zablul Tareq ${ }^{3 *}$ and Shamsun Nahar \\ Begum $^{4}$
}

${ }^{1}$ Dept .of Agricultural Extension, Ministry of Agriculture, Dhaka-1215

2Dept. of Biotechnology, Bangladesh Agricultural University, Mymensingh

3 Jute Agriculture Experimental Station, Bangladesh Jute Research Institute, Manikganj

${ }^{4}$ Biotechnology Division, Bangladesh Institute of Nuclear Agriculture, Mymensingh, Bangladesh

$\triangle$ Corresponding author*: zablulbarj [@] gmail.com

Article Received: 11.08.17; Revised: 14.12.17; Published online: 31 December 2017.

\begin{abstract}
Embryogenic calli from mature seeds of four indica rice genotypes were used to observe their regeneration potentiality and establish a suitable in vitro plantlet regeneration protocol. MS medium supplemented with different phytohormone combinations were used to observe the callus induction ability of the explant. The highest callus induction (73.19\%), biggest size of callus $(3.133 \mathrm{~mm})$ and higher callus weight $(0.7167 \mathrm{~g})$ were observed in Binadhan-6 in MS medium supplemented with $1.0 \mathrm{mg} \mathrm{L}^{-1} 2,4-D$ over all the genotypes and MS medium supplemented with 1.5 $m g L^{-1}$ 2,4-D was the best over all the treatments (66.83\%). Among the phytohormone combinations, $M S+8 \mathrm{mg} \mathrm{L}^{-1}$ Kinetin $+0.5 \mathrm{mg} \mathrm{L}^{-1} \mathrm{NAA}$ showed the highest shoot regeneration (50.67\%) and shoot length $(13.7 \mathrm{~cm})$. Among the genotypes, Binadhan- 6 was highly responsive to shoot regeneration (55.83\%). The best root formation from regenerants (87.889\%), maximum number of roots per plant (20) and the highest length $(4.467 \mathrm{~cm})$ of roots were in MS media supplemented with $0.5 \mathrm{mg} \mathrm{L}^{-1}$ IAA in Binadhan-6. In pot and soil, Binadhan-6 showed the highest survival rate of the plantlet $91.30 \%$ and $85 \%$, respectively. This callus induction and in vitro regeneration protocol will be widely applicable for the tissue culture of indica rice.
\end{abstract}

Key Words: Rice, In vitro callus induction, Protocol development and plant regeneration

Cite Article: Miah, K., Hossen, B., Haque, M. S., Tareq, M. Z. and Begum, S. N. (2017). In vitro regeneration protocol for indica rice genotypes. Journal of Bioscience and Agriculture Research,16(01), 1314-1323. Crossref: https://doi.org/10.18801/jbar.160117.163

Article distributed under terms of a Creative Common Attribution 4.0 International License.

\section{Introduction}

Rice (Oryza sativa L.) a cereal grain, is the most widely consumed staple food for a large part of the world's human population. Rice is the agricultural commodity with the third-the highest worldwide production, after sugarcane and maize (FAOSTAT, 2012). In Bangladesh, rice is occupying 11.0 million ha (BBS, 2013) almost 13 million farm families of the country grow rice which has remained almost stable over the past three decades (BRRI, 2013). 
Global population is increasing very rapidly. Loss in crop production could lead to hunger and famine, especially in the developing county like Bangladesh. For this, it requires research to produce more food efficiently. This improvement can possible be achieved by creating genetic variability. To bring genetic variability, conventional breeding method is not sufficient to elevate rice productivity and yields (Bhuiyan and Karim, 2002). Although conventional breeding will continue to play a major role in increasing crop yield, laboratory based techniques, such as genetic transformation to introduce novel genes into crop plants, will be essential in complementing existing technologies (Ingram et al., 2001). Plant tissue culture technique had made possible to produce genetic variation that are raise crop yield and quality, and that can help to substantially food security (Vasil, 1998). The objective of the experiment was to develop suitable protocol for callus induction and in vitro plant regeneration.

\section{Materials and Methods}

Binadhan-5, Binadhan-6, BRRI dhan32 and Basmati 370 were used as plant material to study different parameters associated with in vitro regeneration of plant. The experiments were carried in 2010 at the Tissue Culture Laboratory, Department of Biotechnology, Bangladesh Agricultural University, Mymensingh and Biotech Laboratory, Bangladesh Institute of Nuclear Agriculture (BINA), Mymensingh.

Culture technique: For callus induction, callus proliferation, shoot regeneration and rooting MS medium (Murashige and Skoog, 1962) was used. The culture techniques were explants culture, partial desiccation, subculture or transfer into regeneration media and rooting employed. Mature embryos attached to endosperm were the main source of explants for embryo culture. The hormones and their respective number of combinations of embryo culture were as follows treatment:

\section{a) For callus induction}

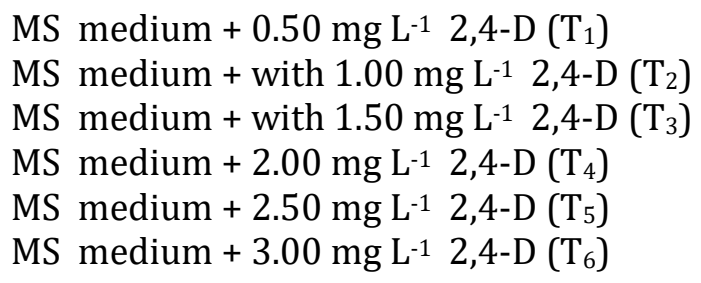

\section{b) For shoot differentiation}

$$
\begin{aligned}
& \text { MS medium + } 2 \mathrm{mg} \mathrm{L}^{-1} \text { Kinetin + } 0.5 \mathrm{mg} \mathrm{L}^{-1} \mathrm{NAA}\left(\mathrm{T}_{1}\right) \\
& \text { MS medium + } 4 \mathrm{mg} \mathrm{L}^{-1} \text { Kinetin }+0.5 \mathrm{mg} \mathrm{L}^{-1} \mathrm{NAA}\left(\mathrm{T}_{2}\right) \\
& \text { MS medium + } 6 \mathrm{mg} \mathrm{L}^{-1} \text { Kinetin }+0.5 \mathrm{mg} \mathrm{L}^{-1} \mathrm{NAA}\left(\mathrm{T}_{3}\right) \\
& \text { MS medium + } 8 \mathrm{mg} \mathrm{L}^{-1} \text { Kinetin }+0.5 \mathrm{mg} \mathrm{L}^{-1} \mathrm{NAA}\left(\mathrm{T}_{4}\right) \\
& \text { MS medium + } 10 \mathrm{mg} \mathrm{L}^{-1} \text { Kinetin }+0.5 \mathrm{mg} \mathrm{L}^{-1} \mathrm{NAA}\left(\mathrm{T}_{5}\right) \\
& \text { MS medium }+12 \mathrm{mg} \mathrm{L}^{-1} \text { Kinetin }+0.5 \mathrm{mg} \mathrm{L}^{-1} \mathrm{NAA}\left(\mathrm{T}_{6}\right)
\end{aligned}
$$

\section{c) For root initiation}

$$
\begin{aligned}
& \text { MS medium + 0.4 mg L-1 IBA }\left(\mathrm{T}_{\mathrm{I}}\right) \\
& \text { MS medium + 0.5 } \mathrm{mg} \mathrm{L}^{-1} \mathrm{IBA}\left(\mathrm{T}_{2}\right) \\
& \text { MS medium + 0.6 } \mathrm{mg} \mathrm{L}^{-1} \mathrm{IBA}\left(\mathrm{T}_{3}\right)
\end{aligned}
$$

Explants culture: Sterilized mature seeds were cultured directly in MS medium supplemented with different concentrations of hormones and sucrose required as per treatment. The culture plates containing explants were placed under fluorescent light in a room with controlled temperature $\left(22 \pm 2^{\circ} \mathrm{C}\right)$ using $16 \mathrm{hrs}$ photoperiod.

Subculture in regeneration media: Three weeks after inoculation, the calli attained convenient size. Then they placed again on freshly prepared sterilized medium containing appropriate hormonal supplements for shoot induction from the calli. The subculture media were MS medium containing different combinations and concentrations of NAA and Kinetin. The subcultured petridishes were again incubated at $22 \pm 2^{\circ} \mathrm{C}$ with $16 \mathrm{hrs}$ photoperiod and mentioned for calli and organogenesis. 
Rooting: The subcultured calli continued to proliferate and differentiated into shoots. When these shoots grew about $2-3 \mathrm{~cm}$ in length, they were separated from each other and again cultured individually on vials or petridishes with freshly prepared root induction medium to induce root. The vials or conical flasks containing plantlets were incubated at $22 \pm 2^{\circ} \mathrm{C}$ with $16 \mathrm{hrs}$ photoperiod.

Data recorded on Callus induction (days of callus initiation, number of explants with callus, size of callus) and plantlet regeneration (days to shoot initiation, number of callus with shoot, number of shoots per callus, average number of roots per plants, percent plant establishment). Complete Randomized Design (CRD) was used in growth room Tissue Culture Laboratory. The analyses of variances for different parameters were performed and means were compared by the Duncan's Multiple Range Test (DMRT) in MSTATC program.

\section{Results and Discussion}

In vitro regeneration protocol of four indica rice genotypes was investigated. Genotypes were accomplished with callus induction, shoot-root development and finally plantlet regeneration.

\section{Callus induction}

Callus initiation started from 05 days of incubation and took about 14 days for the completion followed by Binadhan-6, Binadhan-5, BRRI dhan32 and Basmati 370 respectably (Plate 01). The average percentage of callus induction was the highest in Binadhan-6 (72\%) and the lowest in Basmati $370(22 \%)$. The highest (94\%) percentage of callus induction was observed in Binadhan-6, $\mathrm{T}_{2}$ (MS + $1.0 \mathrm{mgL}^{-1} 2,4-\mathrm{D}$ ) and the lowest (12\%) percentage of callus induction was observed in $\mathrm{T}_{6}$ (MS $+3.0 \mathrm{mgL}^{-1}$ 2,4-D) (Table 01). Callusing was also the highest (66.83\%) in $\mathrm{T}_{3}\left(\mathrm{MS}+1.5 \mathrm{mgL}^{-1}\right.$ 2,4-D) and the lowest (20.29\%) in $\mathrm{T}_{6}$ (MS + $3.0 \mathrm{mgL}^{-1}$ 2,4-D). Hidayat et. al., (2007) reported that maximum callus formation (62.5\%) was recorded for Basmati-385, followed by Basmati-370 (55.55\%) when seeds were cultured on MS medium supplemented with 2,4-D@ $2.0 \mathrm{mgL}^{-1}$.

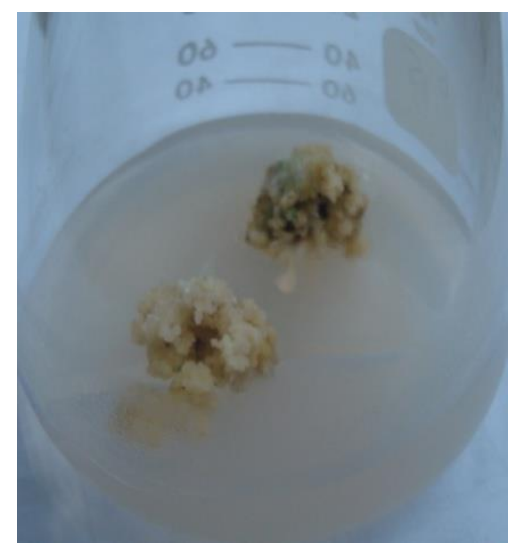

Binadhan-6 (the highest callus)

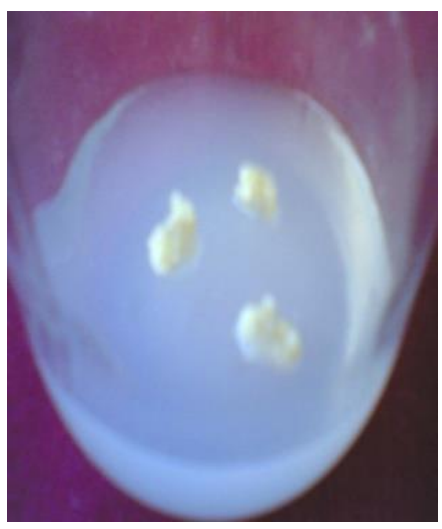

Basmati 370 (the lowest callus)

\section{Plate 01. Callus of four indica rice genotypes from mature embryos with desiccation treatment (subculture).}


Table 01. Effect of different treatment combinations for callus induction from mature embryos of four indica rice variety

\begin{tabular}{|c|c|c|c|c|c|}
\hline Treatment & Genotype & $\begin{array}{c}\text { No. of } \\
\text { explants } \\
\text { inoculated }\end{array}$ & $\begin{array}{l}\text { Explants no. } \\
\text { producing } \\
\text { callus }\end{array}$ & $\begin{array}{l}\% \text { callus } \\
\text { induction }\end{array}$ & $\begin{array}{c}\text { Days required } \\
\text { for callus } \\
\text { induction }\end{array}$ \\
\hline \multirow{4}{*}{$\begin{array}{l}\mathrm{MS}+0.5 \mathrm{mgL}^{-} \\
12,4-\mathrm{D}\left(\mathrm{T}_{1}\right)\end{array}$} & \multirow{4}{*}{$\begin{array}{l}\text { Binadhan-5 } \\
\text { Binadhan-6 } \\
\text { Basmati } 370 \\
\text { BRRI dhan } \\
32\end{array}$} & 50 & 28 & 56 & $9-14$ \\
\hline & & 50 & 42 & 84 & $6-8$ \\
\hline & & 50 & 8 & 16 & $11-15$ \\
\hline & & 50 & 27 & 54 & $6-9$ \\
\hline \multirow{4}{*}{$\begin{array}{l}\mathrm{MS}+1.0 \mathrm{mgL}- \\
12,4-\mathrm{D}\left(\mathrm{T}_{2}\right)\end{array}$} & \multirow{4}{*}{$\begin{array}{l}\text { Binadhan-5 } \\
\text { Binadhan-6 } \\
\text { Basmati } 370 \\
\text { BRRI dhan } \\
32\end{array}$} & 50 & 31 & 62 & $11-12$ \\
\hline & & 50 & 47 & 94 & $7-8$ \\
\hline & & 50 & 13 & 26 & $13-15$ \\
\hline & & 50 & 30 & 60 & $5-7$ \\
\hline \multirow{4}{*}{$\begin{array}{l}\mathrm{MS}+1.5 \mathrm{mgL}- \\
12,4-\mathrm{D}\left(\mathrm{T}_{3}\right)\end{array}$} & \multirow{4}{*}{$\begin{array}{l}\text { Binadhan-5 } \\
\text { Binadhan-6 } \\
\text { Basmati } 370 \\
\text { BRRI dhan } \\
32 \\
\end{array}$} & 50 & 36 & 72 & $10-11$ \\
\hline & & 50 & 42 & 84 & $8-9$ \\
\hline & & 50 & 13 & 26 & $11-14$ \\
\hline & & 50 & 37 & 74 & $5-6$ \\
\hline \multirow{4}{*}{$\begin{array}{l}\mathrm{MS}+2.0 \mathrm{mgL}^{-} \\
12,4-\mathrm{D}\left(\mathrm{T}_{4}\right)\end{array}$} & \multirow{4}{*}{$\begin{array}{l}\text { Binadhan-5 } \\
\text { Binadhan-6 } \\
\text { Basmati } 370 \\
\text { BRRI dhan } \\
32\end{array}$} & 50 & 35 & 70 & $11-14$ \\
\hline & & 50 & 37 & 74 & $8-9$ \\
\hline & & 50 & 19 & 38 & $14-16$ \\
\hline & & 50 & 29 & 58 & $7-10$ \\
\hline \multirow{4}{*}{$\begin{array}{l}\mathrm{MS}+2.5 \mathrm{mgL}- \\
12,4-\mathrm{D}\left(\mathrm{T}_{5}\right)\end{array}$} & \multirow{4}{*}{$\begin{array}{l}\text { Binadhan-5 } \\
\text { Binadhan-6 } \\
\text { Basmati } 370 \\
\text { BRRI dhan } \\
32\end{array}$} & 50 & 23 & 46 & $12-14$ \\
\hline & & 50 & 29 & 58 & 8-10 \\
\hline & & 50 & 7 & 14 & $14-15$ \\
\hline & & 50 & 17 & 34 & $8-10$ \\
\hline \multirow{4}{*}{$\begin{array}{l}\mathrm{MS}+3.0 \mathrm{mgL}- \\
12,4-\mathrm{D}\left(\mathrm{T}_{6}\right)\end{array}$} & \multirow{4}{*}{$\begin{array}{l}\text { Binadhan-5 } \\
\text { Binadhan-6 } \\
\text { Basmati } 370 \\
\text { BRRI dhan } \\
32\end{array}$} & 50 & 8 & 16 & $13-15$ \\
\hline & & 50 & 19 & 38 & 8-11 \\
\hline & & 50 & 6 & 12 & $13-15$ \\
\hline & & 50 & 8 & 16 & 7-11 \\
\hline \multirow{4}{*}{ Average } & Binadhan-5 & 50 & 26.83 & 53.67 & - \\
\hline & Binadhan-6 & 50 & 36 & 72 & - \\
\hline & Basmati 370 & 50 & 11 & 22 & - \\
\hline & BRRI dhan & 50 & 24.67 & 49.33 & - \\
\hline
\end{tabular}

\section{Effect of different treatments on callus characters}

Mean square values of six different combinations of treatments were found statistically significant for the callus characters such as size and weight of callus. $\mathrm{T}_{3}\left(\mathrm{MS}+1.5 \mathrm{mgL}^{-1} 2,4-\mathrm{D}\right.$ ) showed the highest percentage $(66.83 \%)$ of callus induction and the lowest $(20.29 \%) \mathrm{T}_{6}\left(\mathrm{MS}+3.0 \mathrm{mgL}^{-1} 2\right.$ 2,4-D). Size of callus was biggest in $\mathrm{T}_{3}$ (MS $+1.5 \mathrm{mgL}^{-1} 2$,4-D) 2.494, closely followed $\mathrm{T}_{2}$ (MS + 1.0mgL-1 2,4-D ) 2.112 and $\mathrm{T}_{4}$ (MS + $2.0 \mathrm{mgL}^{-1}$ 2,4-D) 2.108 which was not statistically significant, but $\mathrm{T}_{5}\left(\mathrm{MS}+2.5 \mathrm{mgL}^{-}\right.$ 1 2,4-D) 1.788 (Figure 01). Smallest (1.315) size of callus was observed in $\mathrm{T}_{6}$ (MS + $3.0 \mathrm{mgL}^{-1} 2,4-\mathrm{D}$ ). Such type of results was obtained by Saharan et al. (2004); Suresh et al. (2001); Chand and Sahrawat (2001). Weight of callus was the highest (0.4508) in $\mathrm{T}_{3}\left(\mathrm{MS}+1.5 \mathrm{mgL}^{-1} 2,4-\mathrm{D}\right)$ and the lowest (0.1817) in $\mathrm{T}_{6}$ (MS + $3.0 \mathrm{mgL}^{-1}$ 2,4-D) which were statistically significant (Figure 01). Similar size and weight of callus was reported by Azira and Bhalla (2000); Dode et al. (2000); Lin and Zhang (2005); Lee et al. (2002). 


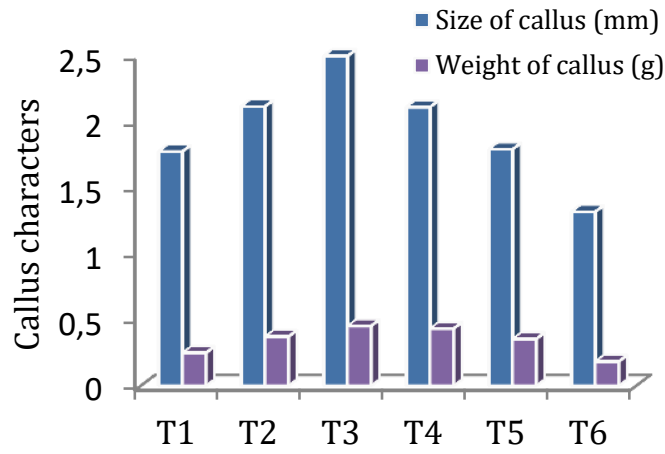

Treatment: $\mathrm{T} 1=\mathrm{MS}+0.5 \mathrm{mgL}^{-1} 2,4-\mathrm{D}, \mathrm{T} 2=\mathrm{MS}+1.0$ $\mathrm{mgL}^{-1} 2,4-\mathrm{D}, \mathrm{T} 3=\mathrm{MS}+1.5 \mathrm{mgL}^{-1} 2,4-\mathrm{D}, \mathrm{T} 4=\mathrm{MS}+2.0$ $\mathrm{mgL}^{-1} 2,4-\mathrm{D}, \mathrm{T} 5=\mathrm{MS}+2.5 \mathrm{mgL}^{-1} 2,4-\mathrm{D}, \mathrm{T} 6=\mathrm{MS}+3.0$ $\mathrm{mgL}^{-1} 2,4-\mathrm{D}$

\section{Figure 01. Response of different treatments on callus characters.}

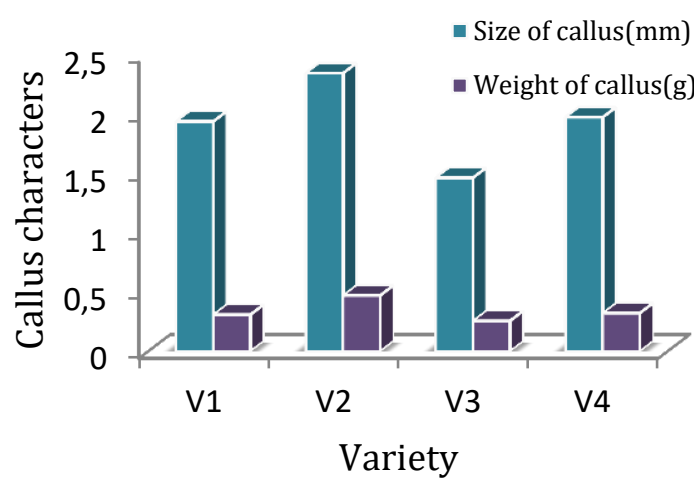

Genotype: V1= Binadhan-5, V2= Binadhan-6, V3= Basmati 370, V4= BRRI dhan32

\section{Figure 02. Response of different genotypes} on callus characters.

\section{Effect of different genotypes on callus characters}

The genotypic mean square values of rice were found statistically significant for all the characters of callus induction. The highest (73.19\%) percentage of callus induction was observed in Binadhan-6 followed by Binadhan-5 (54.83\%), BRRI dhan32 (51.67\%) and the lowest (23.44\%) in Basmati 370 which were statistically significant (Figure 02). Size of callus was biggest (2.347) in Binadhan-6, closely followed BRRI dhan32 (1.975), Binadhan-5 (1.939) which was not statistically significant, Basmati 370 showed smallest (1.464) size of callus. Binadhan- 6 showed much 0.4708 weight of callus followed by BRRI dhan32 (0.3217), Binadhan-5 (0.3081) and Basmati 370 showed the lowest (0.2558) weight of callus which were statistically significant. YuMei et al. (2006) also found this type of result.

\section{Effects of Genotype x Treatment interaction on callus characters}

Among the four genotypes Binadhan-6 showed the highest (95.0\%) percentage of callus induction in $\mathrm{T}_{2}$ (MS $+1.0 \mathrm{mgL}^{-1}$ 2,4-D). The lowest percent of callus induction was observed in Basmati 370 , BRRI dhan32, Binadhan-5 and Binadhan- 6 11, 15, 21.33, and 33.83\% respectively, in $\mathrm{T}_{6}$ (MS +3.0 $\mathrm{mgL}^{-1}$ 2,4-D). Biggest size of callus (3.133) was observed in Binadhan-6 on the interaction of $\mathrm{T}_{2}$ (MS + $1.0 \mathrm{mgL}^{-1}$ 2,4-D), followed by BRRI dhan32, Binadhan-5 and Basmati 370 2.867, 2.583 and 1.758 respectively on the interaction of $\mathrm{T}_{3}$ (MS $\left.+1.5 \mathrm{mgL}^{-1} 2,4-\mathrm{D}\right)$. Smallest size of callus $(1.650,1.342$, 1.267 and 1.000) was found in Binadhan-6, BRRI dhan32, Binadhan-5 and Basmati 370 respectively on the interaction of $\mathrm{T}_{6}$ (MS + 3.0 $\left.\mathrm{mgL}^{-1} 2,4-\mathrm{D}\right)$. Higher weight of callus (0.7167) was observed in Binadhan -6 on the interaction of $\mathrm{T}_{2}\left(\mathrm{MS}+1.0 \mathrm{mgL}^{-1} 2,4-\mathrm{D}\right)$. These findings compared the findings of Azira and Bhalla (2000), Dode et al. (2000) in callus induction and Lin and Zhang (2005) and Lee et al. (2002) in size of callus.

\section{Effects of different treatments on shoot regeneration}

The various levels of Kinetin showed significant variations for percent of shoot regeneration from callus presented on. It was observed that among the treatments tested, number of shoots per explants showed maximum (17.67) on $\mathrm{T}_{4}$ (MS $+8 \mathrm{mgL}^{-1} \mathrm{Kn}+0.5 \mathrm{mgL}^{-1} \mathrm{NAA}$ ) and minimum (3.083) on $\mathrm{T}_{6}$ (MS + $12 \mathrm{mgL}^{-1} \mathrm{Kn}+0.5 \mathrm{mgL}^{-1} \mathrm{NAA}$ ) (Figure 03). Similar trends of result were found by Saharan et al. (2004) and Chand and Sahrawat (2001). The length of shoots was the highest (13.70) on $\mathrm{T}_{4}\left(\mathrm{MS}+8 \mathrm{mgL}^{-1}\right.$ $\left.\mathrm{Kn}+0.5 \mathrm{mgL}^{-1} \mathrm{NAA}\right)$ and the lowest (4.358) on $\mathrm{T}_{6}\left(\mathrm{MS}+12 \mathrm{mgL}^{-1} \mathrm{Kn}+0.5 \mathrm{mgL}^{-1} \mathrm{NAA}\right)$. 


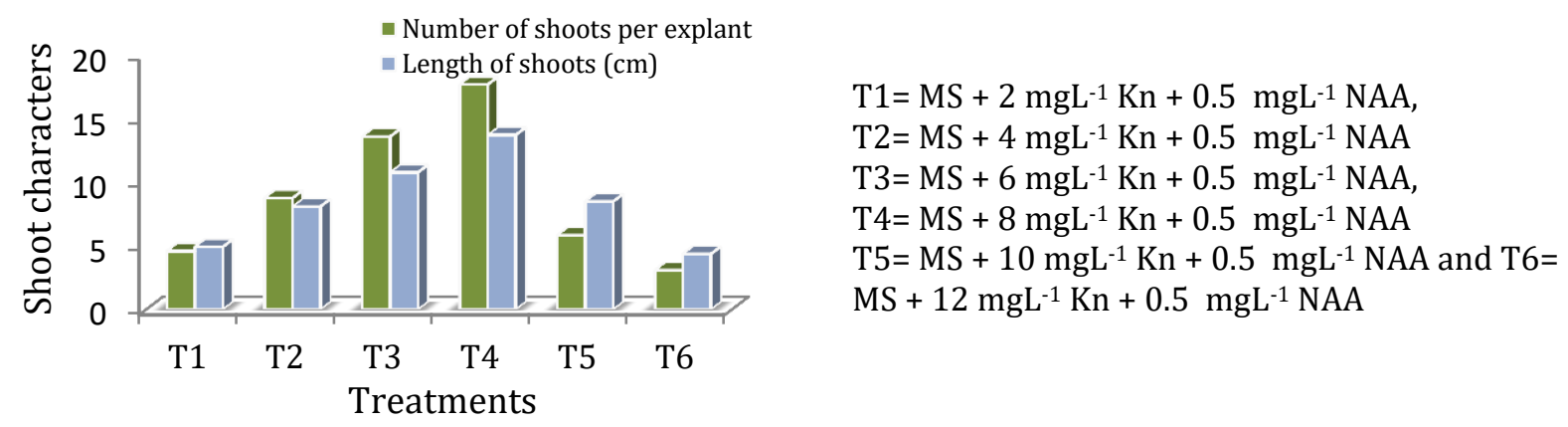

Figure 03. Response of different treatment on

\section{Effects of genotype on shoot regeneration}

Out of four genotypes tested Binadhan-6 showed maximum (16.89) number of shoot callus ${ }^{-1}$ and minimum (2.778) by Basmati 370 (Table 02). Length of shoot was observed the highest in Binadhan-6 (11.64) and the lowest (5.078) by Basmati 370. Hoque and Mansfield (2004) reported that plant regeneration was influenced by genotype.

\section{Effects of treatment $x$ genotype interactions on shoot regeneration}

The highest percentage of shoot regeneration was obtained by Binadhan-6 (79.33) on $\mathrm{T}_{4}\left(\mathrm{MS}+8 \mathrm{mgL}^{-1}\right.$ $\left.\mathrm{Kn}+0.5 \mathrm{mgL}^{-1} \mathrm{NAA}\right)$ and the lowest by Basmati $370(8 \%)$ on $\mathrm{T}_{1}\left(\mathrm{MS}+2 \mathrm{mgL}^{-1} \mathrm{Kn}+0.5 \mathrm{mgL}^{-1} \mathrm{NAA}\right)$ and $\mathrm{T}_{6}$ (MS $+12 \mathrm{mgL}^{-1} \mathrm{Kn}+0.5 \mathrm{mgL}^{-1} \mathrm{NAA}$ ) (Table 03). Out of four genotypes Binadhan- 6 showed the highest (34.00) number of shoots per explants. It was observed that Basmati 370 showed the lowest number of shoot callus (0.667) followed by Binadhan-5 (2.333), BRRI dhan32 (4.333) and Binadhan-6 (5.000) on $\mathrm{T}_{6}$ (MS $+12 \mathrm{mgL}^{-1} \mathrm{Kn}+0.5 \mathrm{mgL}^{-1} \mathrm{NAA}$ ). This finding is similar to Visarada et al. (2002) result.

\section{Effect of genotypes on root induction}

Mean square values of four genotypes were found statistically significant for per cent root induction, number of root/plant and length of roots (Figure 04). The highest (87.88\%) percentage of root induction was observed by Binadhan -6 and the lowest $(40.00 \%)$ by Basmati 370 . Binadhan- 6 showed the highest (4.000) length of roots and Basmati 370 showed the lowest (1.778).

\section{Effects of treatment on root induction}

The various levels of IAA tested on root regeneration showed significant variation for percentage of root induction, number of roots per plant and length (Figure 05). The highest (84.17) percentage of root induction was found on $\mathrm{T}_{2}\left(\mathrm{MS}+0.5 \mathrm{mgL}^{-1} \mathrm{IAA}\right)$ followed by $\mathrm{T}_{1}(\mathrm{MS}+0.3$ $\mathrm{mgL}^{-1} \mathrm{IAA}$ ) $64.75 \%$ and $\mathrm{T}_{3}$ (MS $\left.+0.7 \mathrm{mgL}^{-1} \mathrm{IAA}\right) 61.42 \%$ but both were statistically similar. $\mathrm{T}_{2}$ (MS + $\left.0.5 \mathrm{mgL}^{-1} \mathrm{IAA}\right)$ showed the highest length (3.408) of roots closely followed by $\mathrm{T}_{3}$ (MS $+0.7 \mathrm{mgL}^{-}$ ${ }^{1}$ IAA) 2.842, $\mathrm{T}_{1}$ (MS + $\left.0.3 \mathrm{mgL}^{-1} \mathrm{IAA}\right) 2.583$.

\section{Effects of genotype $x$ treatment interactions on root induction}

Percent of root induction was found the highest (97.67\%) by Binadhan- 6 onT 2 (MS +0.5 mgL $^{-1}$ IAA) and the lowest $(29.00 \%)$ by Basmati 370 onT $_{1}\left(\mathrm{MS}+0.3 \mathrm{mgL}^{-1}\right.$ IAA. There is no significant difference between the interaction on genotypes and treatment (Table 04). Maximum number of roots per plant (20) was observed on $\mathrm{T}_{2}$ (MS $+0.5 \mathrm{mgL}^{-1} \mathrm{IAA}$ ) in genotype of Binadhan- 6 and the lowest (4.333) on $\mathrm{T}_{3}$ (MS + 0.7 $\mathrm{mgL}^{-1}$ ) in Basmati 370 . 
Table 02. Effect of genotypes on shoot regeneration

\begin{tabular}{llll}
\hline Genotypes & $\begin{array}{l}\text { No. of explants } \\
\text { Showing shoot } \\
\text { regeneration }\end{array}$ & $\begin{array}{l}\text { Percent of shoot } \\
\text { regeneration }\end{array}$ & $\begin{array}{l}\text { Days required for } \\
\text { shoot regeneration }\end{array}$ \\
\hline Binadhan-5 & $12.61 \mathrm{~b}$ & $41.83 \mathrm{~b}$ & 16.500 \\
Binadhan-6 & $16.89 \mathrm{a}$ & $55.83 \mathrm{a}$ & 13.500 \\
Basmati 370 & $4.833 \mathrm{~d}$ & $16.11 \mathrm{~d}$ & 19.500 \\
BRRI dhan32 & $10.39 \mathrm{c}$ & $34.22 \mathrm{c}$ & 14.000 \\
\hline$C V(\%)$ & 9.07 & 9.45 & 5.59 \\
$L S D_{(0.05)}$ & 0.6795 & 2.343 & 0.6132 \\
\hline
\end{tabular}

In the column figures followed by same letter(s) do not differ significantly

Table 03. Effects of treatment $x$ genotype interactions on shoot regeneration of four indica rice Genotypes

\begin{tabular}{|c|c|c|c|c|c|c|}
\hline Genotypes & Treatment & $\begin{array}{l}\text { Explants } \\
\text { Showing } \\
\text { shoot } \\
\text { regeneration }\end{array}$ & $\begin{array}{l}\text { Percent of } \\
\text { shoot } \\
\text { regenerati } \\
\text { on } \\
\end{array}$ & $\begin{array}{l}\text { Days required } \\
\text { shoot } \\
\text { regeneration }\end{array}$ & $\begin{array}{c}\text { Number of } \\
\text { shoot per } \\
\text { explant }\end{array}$ & $\begin{array}{l}\text { Length of } \\
\text { shoot } \\
\text { (cm) }\end{array}$ \\
\hline \multirow{4}{*}{$\begin{array}{l}\mathrm{MS}+2 \mathrm{mgL}^{-1} \\
\mathrm{Kn}+0.5 \\
\mathrm{mgL}^{-1} \mathrm{NAA} \\
\left(\mathrm{T}_{1}\right)\end{array}$} & Binadhan-5 & $9.000 \mathrm{hi}$ & $29.67 \mathrm{hi}$ & 19.33 & $4.333 \mathrm{hij}$ & $5.000 \mathrm{jkl}$ \\
\hline & Binadhan-6 & $12.33 \mathrm{ef}$ & $41.00 \mathrm{ef}$ & 11.67 & $8.333 \mathrm{fg}$ & $6.833 \mathrm{gh}$ \\
\hline & Basmati 370 & $2.333 \mathrm{~m}$ & $8.000 \mathrm{~m}$ & 24.00 & $1.667 \mathrm{jk}$ & 3.167 no \\
\hline & BRRI dhan32 & $7.667 \mathrm{ij}$ & $25.00 \mathrm{ij}$ & 23.00 & $4.000 \mathrm{ijk}$ & $4.667 \mathrm{klm}$ \\
\hline \multirow{4}{*}{ 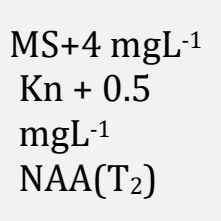 } & Binadhan-5 & $14.00 \mathrm{de}$ & 46.33 de & 16.00 & $4.667 \mathrm{hij}$ & $7.833 \mathrm{fg}$ \\
\hline & Binadhan-6 & $15.00 \mathrm{~cd}$ & $49.67 \mathrm{~cd}$ & 9.333 & $20.00 \mathrm{c}$ & $11.23 \mathrm{~d}$ \\
\hline & Basmati 370 & $5.000 \mathrm{kl}$ & $16.67 \mathrm{kl}$ & 20.67 & $2.667 \mathrm{jk}$ & $5.600 \mathrm{ijk}$ \\
\hline & BRRI dhan32 & $11.33 \mathrm{fg}$ & $37.33 \mathrm{fg}$ & 20.00 & $7.667 \mathrm{fgh}$ & $7.667 \mathrm{fg}$ \\
\hline \multirow{4}{*}{$\begin{array}{l}\mathrm{MS}+6 \mathrm{mgL}^{-1} \\
\mathrm{Kn}+0.5 \\
\mathrm{mgL}^{-1} \mathrm{NAA} \\
\left(\mathrm{T}_{3}\right)\end{array}$} & Binadhan-5 & $16.00 \mathrm{c}$ & $53.00 \mathrm{c}$ & 11.33 & $15.33 \mathrm{~d}$ & $13.53 \mathrm{c}$ \\
\hline & Binadhan-6 & $18.00 \mathrm{~b}$ & $59.33 \mathrm{~b}$ & 8.333 & $27.67 \mathrm{~b}$ & $15.43 \mathrm{~b}$ \\
\hline & Basmati 370 & $6.667 \mathrm{jk}$ & $22.00 \mathrm{jk}$ & 17.00 & 4.667 hij & $5.833 \mathrm{ij}$ \\
\hline & BRRI dhan32 & $12.33 \mathrm{ef}$ & 40.67 ef & 17.33 & 6.667 fghi & $8.300 \mathrm{f}$ \\
\hline \multirow{4}{*}{$\begin{array}{l}\mathrm{MS}+8 \mathrm{mgL}^{-} \\
{ }^{1} \mathrm{Kn}+0.5 \\
\mathrm{mgL}^{-1} \mathrm{NAA} \\
\left(\mathrm{T}_{4}\right)\end{array}$} & Binadhan-5 & $14.00 \mathrm{de}$ & $46.33 \mathrm{de}$ & 16.33 & $11.67 \mathrm{e}$ & $11.90 \mathrm{~d}$ \\
\hline & Binadhan-6 & $24.00 \mathrm{a}$ & $79.33 \mathrm{a}$ & 6.667 & $34.00 \mathrm{a}$ & $19.83 \mathrm{a}$ \\
\hline & Basmati 370 & $8.000 \mathrm{ij}$ & $26.33 \mathrm{ij}$ & 19.67 & $3.667 \mathrm{ijk}$ & $7.533 \mathrm{fg}$ \\
\hline & BRRI dhan32 & $15.33 \mathrm{~cd}$ & $50.67 \mathrm{~cd}$ & 13.33 & $21.33 \mathrm{c}$ & $15.53 \mathrm{~b}$ \\
\hline \multirow{4}{*}{$\begin{array}{l}\mathrm{MS}+10 \\
\mathrm{mgL}^{-1} \mathrm{Kn}+ \\
0.5 \mathrm{mgL}^{-1} \\
\mathrm{NAA}\left(\mathrm{T}_{5}\right)\end{array}$} & Binadhan-5 & $12.00 \mathrm{f}$ & 40.67 ef & 20.00 & $3.667 \mathrm{ijk}$ & $9.767 \mathrm{e}$ \\
\hline & Binadhan-6 & $18.00 \mathrm{~b}$ & $59.33 \mathrm{~b}$ & 11.00 & 6.333 ghi & 10.17 e \\
\hline & Basmati 370 & $4.667 \mathrm{l}$ & $15.67 \mathrm{l}$ & 23.33 & $3.333 \mathrm{ijk}$ & $5.700 \mathrm{ij}$ \\
\hline & BRRI dhan32 & $10.00 \mathrm{gh}$ & $33.00 \mathrm{gh}$ & 21.33 & $10.00 \mathrm{ef}$ & $8.300 \mathrm{f}$ \\
\hline \multirow{6}{*}{$\begin{array}{l}\mathrm{MS}+12 \mathrm{mgL}^{-} \\
{ }^{1} \mathrm{Kn}+0.5 \\
\mathrm{mgL}^{-1} \mathrm{NAA} \\
\left(\mathrm{T}_{6}\right)\end{array}$} & Binadhan-5 & $10.67 \mathrm{fgh}$ & $35.00 \mathrm{fgh}$ & 23.33 & $2.333 \mathrm{jk}$ & $3.867 \mathrm{mn}$ \\
\hline & Binadhan-6 & $14.00 \mathrm{de}$ & $46.33 \mathrm{de}$ & 13.00 & 5.000 ghij & $6.367 \mathrm{hi}$ \\
\hline & Basmati 370 & $2.333 \mathrm{~m}$ & $8.000 \mathrm{~m}$ & 24.67 & $0.667 \mathrm{k}$ & 2.633 o \\
\hline & BRRI dhan32 & $5.667 \mathrm{kl}$ & $18.67 \mathrm{kl}$ & 25.67 & $4.333 \mathrm{hij}$ & $4.567 \mathrm{~lm}$ \\
\hline & $C V(\%)$ & 9.07 & 9.45 & 5.59 & 21.02 & 6.71 \\
\hline & $L S D_{(0.05)}$ & 1.665 & 5.739 & 1.876 & 3.077 & 0.9228 \\
\hline
\end{tabular}

In the column figures followed by same letter(s) do not differ significantly. 


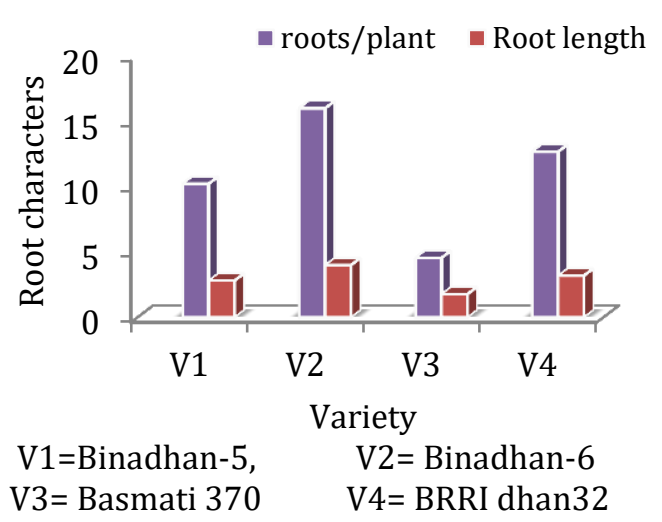

Figure 04. Performance of four indica rice genotypes on root induction.

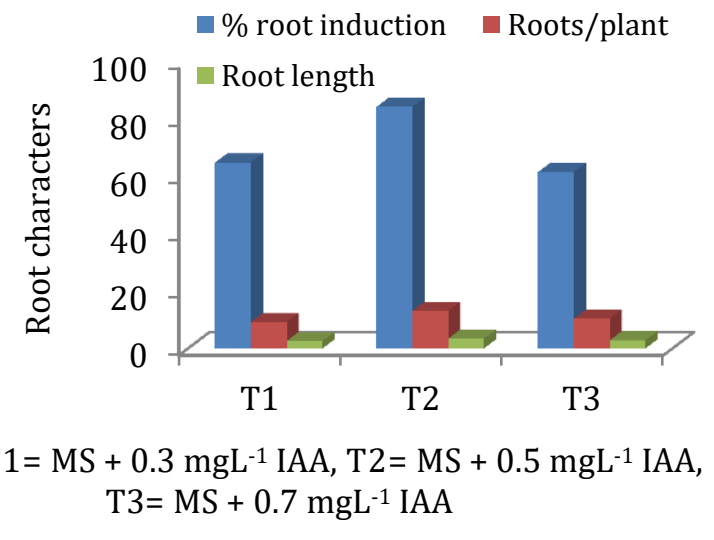

Figure 05. Response of different treatments on root induction of four rice genotypes.

Table 04. Effects of genotype $x$ treatment interactions on root induction of four indica rice Genotypes

\begin{tabular}{llccc}
\hline Genotypes & Treatment & $\begin{array}{c}\text { Percent of root } \\
\text { induction }\end{array}$ & $\begin{array}{c}\text { Number of } \\
\text { roots per plant }\end{array}$ & $\begin{array}{c}\text { Length of } \\
\text { roots (cm) }\end{array}$ \\
\hline Binadhan-5 & MS + 0.3 mgL-1 IAA $\left(\mathrm{T}_{1}\right)$ & $70.67 \mathrm{def}$ & $8.333 \mathrm{de}$ & $2.433 \mathrm{e}$ \\
& $\mathrm{MS}+0.5 \mathrm{mgL}^{-1} \mathrm{IAA}\left(\mathrm{T}_{2}\right)$ & $88.33 \mathrm{ab}$ & $12.67 \mathrm{bc}$ & $3.200 \mathrm{c}$ \\
& $\mathrm{MS}+0.7 \mathrm{mgL}^{-1} \mathrm{IAA}\left(\mathrm{T}_{3}\right)$ & $62.00 \mathrm{fg}$ & $9.667 \mathrm{~cd}$ & $2.800 \mathrm{~d}$ \\
Binadhan-6 & $\mathrm{MS}+0.3 \mathrm{mgL}^{-1} \mathrm{IAA}\left(\mathrm{T}_{1}\right)$ & $84.00 \mathrm{bc}$ & $13.33 \mathrm{bc}$ & $3.700 \mathrm{~b}$ \\
& $\mathrm{MS}+0.5 \mathrm{mgL}^{-1} \mathrm{IAA}\left(\mathrm{T}_{2}\right)$ & $97.67 \mathrm{a}$ & $20.00 \mathrm{a}$ & $4.467 \mathrm{a}$ \\
& $\mathrm{MS}+0.7 \mathrm{mgL}^{-1} \mathrm{IAA}\left(\mathrm{T}_{3}\right)$ & $82.00 \mathrm{bcd}$ & $14.67 \mathrm{~b}$ & $3.833 \mathrm{~b}$ \\
Basmati 370 & $\mathrm{MS}+0.3 \mathrm{mgL}^{-1} \mathrm{IAA}\left(\mathrm{T}_{1}\right)$ & $29.00 \mathrm{~h}$ & $4.000 \mathrm{f}$ & $1.433 \mathrm{f}$ \\
& $\mathrm{MS}+0.5 \mathrm{mgL}^{-1} \mathrm{IAA}\left(\mathrm{T}_{2}\right)$ & $57.67 \mathrm{~g}$ & $5.333 \mathrm{ef}$ & $2.267 \mathrm{e}$ \\
BRRI dhan32 & $\mathrm{MS}+0.7 \mathrm{mgL}^{-1} \mathrm{IAA}\left(\mathrm{T}_{3}\right)$ & $33.33 \mathrm{~h}$ & $4.333 \mathrm{f}$ & $1.633 \mathrm{f}$ \\
& $\mathrm{MS}+0.3 \mathrm{mgL}^{-1} \mathrm{IAA}\left(\mathrm{T}_{1}\right)$ & $75.33 \mathrm{cde}$ & $11.00 \mathrm{bcd}$ & $2.767 \mathrm{~d}$ \\
& $\mathrm{MS}+0.5 \mathrm{mgL}^{-1} \mathrm{IAA}\left(\mathrm{T}_{2}\right)$ & $93.00 \mathrm{ab}$ & $14.33 \mathrm{~b}$ & $3.700 \mathrm{~b}$ \\
& $\mathrm{MS}+0.7 \mathrm{mgL}^{-1} \mathrm{IAA}\left(\mathrm{T}_{3}\right)$ & $68.33 \mathrm{efg}$ & $12.67 \mathrm{bc}$ & $3.100 \mathrm{c}$ \\
\hline$C V(\%)$ & & 6.96 & 6.51 & 6.45 \\
$L S D_{(0.05)}$ & & 11.840 & 3.556 & 0.2064 \\
\hline
\end{tabular}

In the column figures followed by same letter(s) do not differ significantly

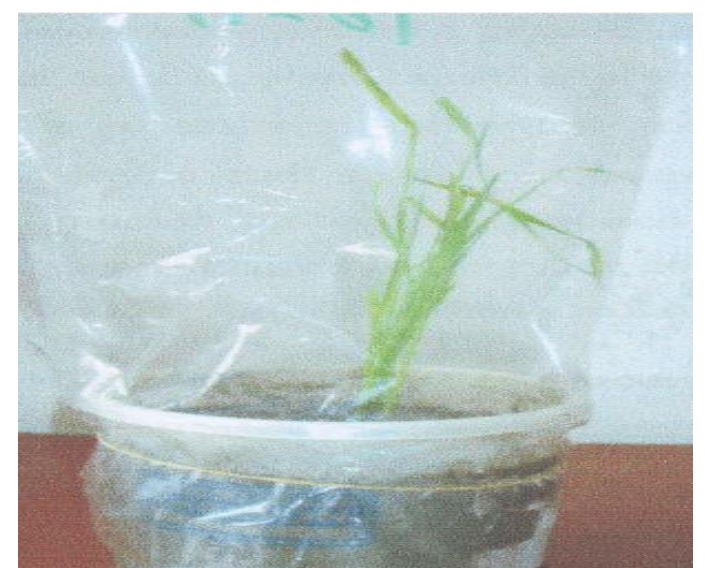

Figure 06. Acclimatized plantlets of Binadhan- 6 in plastic pot covered with polythene bag.

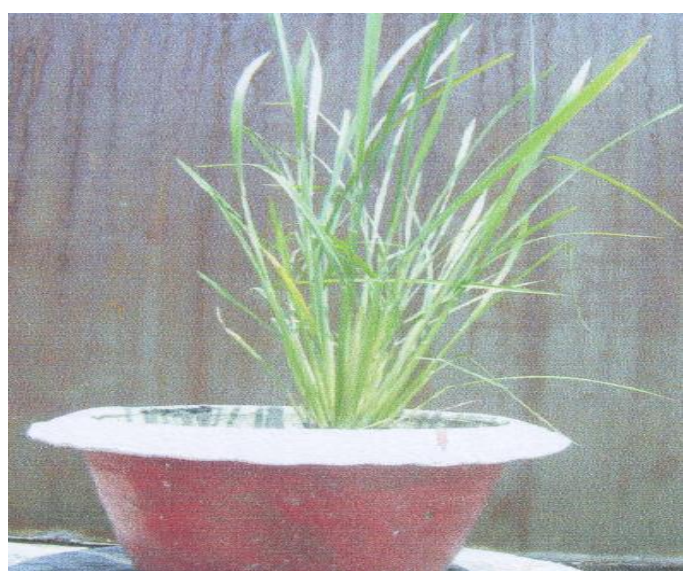

Figure 07. Established plantlets of Binadhan- 6 in earthen pot. 


\section{Establishment of plantlets}

The small plantlets, after sufficient development in root system, were taken out from the culture vessels without causing any damage to roots. The plantlets of Binadhan-6, BRRI dhan32, Binadhan-5, and Basmati 370 was 91.30, 85, 80 and 53.33\%, respectively in pot and Binadhan-6, Binadhan-5, BRRI dhan32 and Basmati 370 was $85,75,72.72$ and $40 \%$, respectively in soil.

\section{Conclusion}

This experiment was carried out to develop a protocol for callus induction and plant regeneration from four indica rice genotypes. The percent callus induction was the highest (72\%) in Binadhan-6 which required minimum (6-7) days for callus initiation and the lowest (22\%) on Basmati 370. Biggest size (3.133) and the highest weight (0.7167) of callus were observed in Binadhan- 6 in $\mathrm{T}_{2}$ (MS + $1.0 \mathrm{mgL}$ 1 2,4-D). After callus formation kinetin and NAA on MS medium were used to observe the shoot regeneration capacity of different calli. Among four genotypes, the maximum (16.89) number of shoot per callus and the highest length of shoot (11.64) were observed in Binadhan-6, while minimum (2.778) number of shoots per callus and the lowest length (5.078) of shoot were obtained in Basmati 370. Among the four treatments, the highest (50.67) percentage of shoot regeneration, maximum (17.67) number of shoots per explants and the highest (13.70) length of shoots were observed on $\mathrm{T}_{4}$ (MS $+8 \mathrm{mg} / \mathrm{L} \mathrm{Kn}+0.5 \mathrm{mg} / \mathrm{L} \mathrm{NAA}$ ), while the lowest (25.92\%) percentage of shoot regeneration was observed in $\mathrm{T}_{1}\left(\mathrm{MS}+2 \mathrm{mgL}^{-1} \mathrm{Kn}+0.5 \mathrm{mgL}^{-1} \mathrm{NAA}\right)$. Minimum (3.083) number of shoots per explants and the lowest (4.358) length of shoots were observed on $\mathrm{T}_{6}\left(\mathrm{MS}+12 \mathrm{mgL}^{-1} \mathrm{Kn}+0.5 \mathrm{mgL}^{-1} \mathrm{NAA}\right)$.

MS medium with different concentrations of IAA $\left(0.3,0.5\right.$ and $\left.0.7 \mathrm{mgL}^{-1}\right)$ were used to observe the rooting responses of regenerated shoots. Among the four genotypes, Binadhan- 6 showed the highest percentage $(87.889 \%)$ of root initiation whereas Basmati 370 showed the lowest $(40 \%)$. The percentage of root initiation was the highest $84.17 \%$ in $\mathrm{T}_{2}$ (MS $+0.5 \mathrm{mgL}^{-1}$ IAA) and the lowest $61.42 \%$ on $\mathrm{T}_{3}$ (MS $\left.+0.7 \mathrm{mgL}^{-1} \mathrm{IAA}\right)$. Maximum number (20.0) of roots per plant and the highest length (4.467) of roots were observed in Binadhan- 6 on $\mathrm{T}_{2}$ (MS $+0.5 \mathrm{mgL}^{-1} \mathrm{IAA}$ ) and minimum number (4.333) of roots per plant and the lowest length (1.633) of roots were observed in Basmati 370 on $\mathrm{T}_{3}\left(\mathrm{MS}+0.7 \mathrm{mgL}^{-1} \mathrm{IAA}\right)$. The survival rate of the plantlet was the highest in Binadhan-6 in the pot (91.30\%) and (85\%) in soil. but BRRI dhan32 showed the highest survival rate (Both in pot and soil). Basmati 370 showed the lowest survival rate of the plantlet $53.33 \%$ and $40 \%$ respectively. This protocol will to help to further study

\section{References}

[1]. Azira, D. and Bhalla, P. L. (2000). Plant regeneration from mature embryo-derived callus of Australian rice (Oryza saliva L.) genotypes. Australian Journal of Agricultural Research 51, 305-312. https://doi.org/10.1071/AR98189

[2]. BBS (2013). Bangladesh Bureau of Statistics. Crop Statistics (Major Crops). Agriculture Wing. Ministry of Planning. Government of the People's Republic of Bangladesh, Dhaka. p. 54.

[3]. Bhuiyan, S. I. and Karim, A. N. M. R. (2002). Rice production in Bangladesh: an overview. Bangladesh Rice Research Institute, Joydebpur, Dhaka. pp. 13-17.

[4]. BRRI (2013). Homepage, Bangladesh Rice Research Institute (BRRI). www.BRRI.org.bd

[5]. Chand, S. and Sahrawat, A. K. (2001). Stimulatory effect of partial desiccation on plant regeneration in indica rice (Oryza sativa L.). Journal of Plant Biochemistry and Biotechnology, 10, 43-47. https://doi.org/10.1007/BF03263105

[6]. Dode, L. B., Goncalves, F. S. M., Oliveira, L. A. A., Vigh, L. L., Magalhees, J. A. M. and Peters, J. P. (2000). Effect of culture media hormonal balance in the induction and proliferation of mature rice seed derived callus. Revista-Cientifica-Rural, 5(2), 27-31.

[7]. FAOSTAT (2012). Food and Agriculture Organization. www.faostat.fao.org

[8]. Hidayat, U., Iltaf, U., Sultan, A. J. and Hamid, R. (2007). Tissue culture techniques for callus induction in rice. Sarhad Journal of Agriculture, 23(1), 82-86.

[9]. Hoque, M. E. and Mansfield, I. W. (2004). Effect of genotype and explants age on callus induction and subsequent plant regeneration from root derived callus rice genotypes. Plant Cell, Tissue and Organ Culture, 78(3), 217-223. 


\section{https://doi.org/10.1023/B:TICU.0000025640.75168.2d}

[10]. Ingram, H. M., Livesey, N. L., Power, J. B. and Davey, M. R. (2001). Genetic transformation of wheat: progress during the 1990s into the millennium, Acta Physiologiae Plantarum, 23(2), 221-239. https://doi.org/10.1007/s11738-001-0012-2

[11]. Lee, K. S., Leon, H. S. and Kim, M. Y. (2002). Optimization of a mature embryo- based In Vitro culture system for high frequency somatic embryogenic callus induction and plant regeneration from japonica rice cultivars. Plant Cell, Tissue and Organ Culture, 71, 9-13.

[12]. Lin, Y. J. and Zhang, Q. (2005). Optimizing the tissue culture condition for high efficiency transformation of indica rice. Plant Cell Reports, 23, 540-547.

https://doi.org/10.1007/s00299-004-0843-6

PMid:15309499

[13]. Murashige, T. and Skoog, F. (1962) A revised medium for rapid growth and bioassay with tobacco tissue cultures. Physiologia Plantarum, 15, 473-497.

https://doi.org/10.1111/j.1399-3054.1962.tb08052.x

[14]. Saharan, V., Yadav, R. C., Yadav, N. R. and Chapagain, B. P. (2004) High frequency plant regeneration from desiccated calli of indica rice (Oryza sativa L.). African Journal of Biotechnology, 3(5), 256-259. https://doi.org/10.5897/AJB2004.000-2047

[15]. Suresh, C., Sahrawat, A. K. and Chand, S. (2001). Stimulatory effect of partial desiccation on plant regeneration in indica rice (Oryza sativa L.). Journal of Plant Biochemistry and Biotechnology, 10(1), 43-47. https://doi.org/10.1007/BF03263105

[16]. Vasil, I. K. (1998). Biotechnology and food security for the $21^{\text {st }}$ century: a real-world perspective. Nature Biotechnology, 16, 399-400.

https://doi.org/10.1038/nbt0598-399

PMid:9592375

[17]. Visarada, K. B. R. S., Sailaja, M. and Sharma, N. P. (2002). Effect of callus induction media on morphology of embryogenic ealli in rice genotypes. Biologia Plantarum, 45(4), 495-502. https://doi.org/10.1023/A:1022323221513

[18]. Visarada, K. B. R. S., Vrjaya Kumar, C. H. M. and Sharma, N. P. (2001). Analysis of variation in tissue culture response for identification of indica rice genotypes suitable for transformation. Journal of Genetics and Breeding, 55, 125-134.

[19]. YuMei, D., FuYou, Y., LingXian, W., ZaiQuan, C. and XingQi, H. (2006). In vitro anther culture of three different genome types of Yunnan wild rice species. Southwest-China-Journal of Agricultural Science, 19(6), 1023-1027.

\section{HOW TO CITE THIS ARTICLE?}

\section{Crossref: https://doi.org/10.18801/jbar.160117.163}

\section{APA (American Psychological Association)}

Miah, K., Hossen, B., Haque, M. S., Tareq, M. Z. and Begum, S. N. (2017). In vitro regeneration protocol for indica rice genotypes. Journal of Bioscience and Agriculture Research,16(01), 1314-1323.

\section{MLA (Modern Language Association)}

Miah, K., Hossen, B., Haque, M. S., Tareq, M. Z. and Begum, S. N. "In vitro regeneration protocol for indica rice genotypes". Journal of Bioscience and Agriculture Research, 16.01(2017): 1314-1323.

\section{Chicago and or Turabian}

Miah, K., Hossen, B., Haque, M. S., Tareq, M. Z. and Begum, S. N. "In vitro regeneration protocol for indica rice genotypes". Journal of Bioscience and Agriculture Research,16 no.01(2017):1314-1323. 